Eduardo Telles Carlos

\title{
Frustum Culling Híbrido Utilizando CPU e GPU
}

Dissertação apresentada ao Programa de Pós-graduação em Informática da PUC-Rio como requisito parcial para obtenção do título de Mestre em Informática.

Orientador : Prof. Alberto Barbosa Raposo

Co-Orientador: Prof. Marcelo Gattass 


\section{Frustum Culling Híbrido Utilizando CPU e GPU}

Dissertação apresentada como requisito parcial para obtenção do grau de Mestre pelo Programa de Pós-graduação em Informática da PUC-Rio. Aprovada pela Comissão Examinadora abaixo assinada.

Prof. Alberto Barbosa Raposo

Orientador

Departamento de Informática - PUC-Rio

Prof. Marcelo Gattass

Co-Orientador

Departamento de Informática - PUC-Rio

Prof. Waldemar Celes Filho

Departamento de Informática — PUC-Rio

D.Sc. Luciano Soares

Departamento de Informática — PUC-Rio

Ph.D. Rodrigo de Toledo

Cenpes - Petrobras

Prof. José Eugênio Leal

Coordenador Setorial do Centro Técnico Científico - PUC-Rio 
Todos os direitos reservados. É proibida a reprodução total ou parcial do trabalho sem autorização da universidade, do autor e do orientador.

\section{Eduardo Telles Carlos}

Graduou-se em Engenharia da computação na PUC-Rio em 2006. Desde 2002 trabalha no Grupo de Tecnologia em Computação Gráfica da universidade (TecGraf) desenvolvendo sistemas de realidade virtual e visualização científica.

Ficha Catalográfica

Carlos, Eduardo Telles

Frustum Culling Híbrido Utilizando CPU e GPU / Eduardo Telles Carlos; orientador: Alberto Barbosa Raposo; co-orientador: Marcelo Gattass. - 2009.

99 f: il. (color.); $30 \mathrm{~cm}$

Dissertação (Mestrado em Informática) - Pontifícia Universidade Católica do Rio de Janeiro, Rio de Janeiro, 2009.

Inclui bibliografia

1. Informática - Teses. 2. Algoritmos de visualização. 3. Descarte de volumes envolventes. 4. GPGPU. 5. Primitivas GPU. I. Raposo, Alberto Barbosa. II. Gattass, Marcelo. III. Pontifícia Universidade Católica do Rio de Janeiro. Departamento de Informática. IV. Título. 
À minha mãe Nilce Telles Carlos, ao meu pai William Braga Carlos e à minha irmã Érica Telles Carlos. Sem o carinho, amor e dedicação deles, este trabalho não seria possível. 


\section{Agradecimentos}

Ao meu orientador Alberto Raposo, pela dedicação, confiança e conselhos dados ao longo de toda a execução deste trabalho.

Ao meu co-orientador Marcelo Gattass pelas sugestões e confiança no meu trabalho.

Aos professores Marcelo Gattass e Waldemar Celes Filho pelas excelentes aulas na graduação e no mestrado.

A meus pais e a minha irmã por tudo que sou hoje.

A minha avó, tios, tias, primos, primas e a minha madrinha pela ajuda, conselhos e amor.

A Pontifícia Universidade Católica do Rio de Janeiro pela excelente formação a mim dada.

Ao Tecgraf pela oportunidade de aprendizado e auxílios concedidos.

A todos os integrantes das equipes do SiVIEP, Environ e demais dentro do Tecgraf pela compreensão, aprendizado e momentos de descontração.

Aos grandes amigos André Luis Pinto Ferreira e Rafael Brito de Farias pelas amizades verdadeiras e apoio nos momentos difíceis.

A Rodrigo Toledo por toda a ajuda dada antes mesmo da definição da área de pesquisa a ser seguida e ajuda no framework das GPU primitives.

A Paulo Ivson Netto pela ajuda nos trabalhos ao longo da graduação, do mestrado e no desenvolvimento deste trabalho.

A equipe de suporte do Tecgraf por terem me ajudado com os problemas de hardware.

A paciência dos integrantes do futebol de domingo pela queda no meu rendimento. 


\section{Resumo}

Carlos, Eduardo Telles; Raposo, Alberto Barbosa; Gattass, Marcelo. Frustum Culling Híbrido Utilizando CPU e GPU. Rio de Janeiro, 2009. 99p. Dissertação de Mestrado — Departamento de Informática, Pontifícia Universidade Católica do Rio de Janeiro.

Um dos problemas mais antigos da computação gráfica tem sido a determinação de visibilidade. Vários algoritmos têm sido desenvolvidos para viabilizar modelos cada vez maiores e detalhados. Dentre estes algoritmos, destaca-se o frustum culling, cujo papel é remover objetos que não sejam visíveis ao observador. Esse algoritmo, muito comum em várias aplicações, vem sofrendo melhorias ao longo dos anos, a fim de acelerar ainda mais a sua execução. Apesar de ser tratado como um problema bem resolvido na computação gráfica, alguns pontos ainda podem ser aperfeiçoados, e novas formas de descarte desenvolvidas. No que se refere aos modelos massivos, necessita-se de algoritmos de alta performance, pois a quantidade de cálculos aumenta significativamente. Este trabalho objetiva avaliar o algoritmo de frustum culling e suas otimizações, com o propósito de obter o melhor algoritmo possível implementado em CPU, além de analisar a influência de cada uma de suas partes em modelos massivos. Com base nessa análise, novas técnicas de frustum culling serão desenvolvidas, utilizando o poder computacional da GPU (Graphics Processing Unit), e comparadas com o resultado obtido apenas pela CPU. Como resultado, será proposta uma forma de frustum culling híbrido, que tentará aproveitar o melhor da CPU e da GPU.

\section{Palavras-chave}

Algoritmos de visualização. Descarte de volumes envolventes. GPGPU. Primitivas GPU. 


\section{Abstract}

Carlos, Eduardo Telles; Raposo, Alberto Barbosa; Gattass, Marcelo. Hybrid Frustum Culling Using CPU and GPU. Rio de Janeiro, 2009. 99p. MSc Dissertation - Departamento de Informática, Pontifícia Universidade Católica do Rio de Janeiro.

The definition of visibility is a classical problem in Computer Graphics. Several algorithms have been developed to enable the visualization of huge and complex models. Among these algorithms, the frustum culling, which plays an important role in this area, is used to remove invisible objects by the observer. Besides being very usual in applications, this algorithm has been improved in order to accelerate its execution. Although being treated as a well-solved problem in Computer Graphics, some points can be enhanced yet, and new forms of culling may be disclosed as well. In massive models, for example, algorithms of high performance are required, since the calculus arises considerably. This work analyses the frustum culling algorithm and its optimizations, aiming to obtain the state-of-the-art algorithm implemented in CPU, as well as explains the influence of each of its steps in massive models. Based on this analysis, new GPU (Graphics Processing Unit) based frustum culling techniques will be developed and compared with the ones using only CPU. As a result, a hybrid frustum culling will be proposed, in order to achieve the best of CPU and GPU processing.

\section{Keywords}

Visualization algorithms. Frustum culling. GPGPU. GPU Primitives. 


\section{Sumário}

$\begin{array}{lll}1 & \text { Introdução } & 13\end{array}$

$\begin{array}{lll}1.1 & \text { Motivação } & 13\end{array}$

\begin{tabular}{lll}
\hline 1.2 & Objetivo da dissertação & 15
\end{tabular}

$\begin{array}{lll}1.3 & \text { Trabalhos relacionados } & 15\end{array}$

1.4 Organização da dissertação 16

$\begin{array}{lll}2 & \text { Algoritmos de Visibilidade } & 17\end{array}$

\begin{tabular}{lll}
\hline 2.1 & Determinação de visibilidade & 17
\end{tabular}

2.2 Classificação dos algoritmos de visibilidade 20

2.3 Pipeline dos algoritmos de visualização 22

$\begin{array}{lll}3 & \text { Frustum culling } & \mathbf{2 6}\end{array}$

\begin{tabular}{lll}
\hline 3.1 & Implementação clássica & 27
\end{tabular}

\begin{tabular}{lll}
\hline 3.2 & Estado da arte & 28
\end{tabular}

3.3 Memória utilizada em CPU 42

3.4 Ambiente de benchmark 43

$\begin{array}{lll}4 & \text { Implementação em CPU } & 48\end{array}$

$\begin{array}{lll}4.1 & \text { Renderização } & 48\end{array}$

\begin{tabular}{|lll}
4.2 & Radar X Planos & 48
\end{tabular}

4.3 Hierarquia $\quad 51$

4.4 Percurso 53

4.5 Otimizações 56

$\begin{array}{lll}4.5 & \text { SIMD } & 58\end{array}$

$\begin{array}{lll}4.7 & \text { Multiprocessamento } & 60\end{array}$

$\begin{array}{lll}4.8 & \text { Pipeline final em CPU } & 64\end{array}$

$\begin{array}{lll}5 & \text { Frustum culling em GPU } & \mathbf{6 5}\end{array}$

$\begin{array}{lll}5.1 & \text { Timeline gpu } & 65\end{array}$

\begin{tabular}{|lll}
5.2 & Gpu primitives & 67
\end{tabular}

$\begin{array}{lll}5.3 & \text { Algoritmos de frustum culling em GPU } & 71\end{array}$

$\begin{array}{lll}5.4 & \text { Memória utilizada em GPU } & 74\end{array}$

$\begin{array}{lll}5.5 & \text { Percurso sem pilha em GPU } & 75\end{array}$

$\begin{array}{lll}5.6 & \text { Implementação } & 76\end{array}$

$\begin{array}{lll}6 & \text { Frustum culling híbrido } & 79\end{array}$

\begin{tabular}{lll}
\hline 6.1 & Heurísticas & 79
\end{tabular}

$\begin{array}{lll}6.2 & \text { Implementação } & 85\end{array}$

$\begin{array}{lll}7 & \text { Conclusão e Trabalhos futuros } & \mathbf{8 9}\end{array}$

$\begin{array}{lll}7.1 & \text { Conclusão } & 89\end{array}$

$\begin{array}{lll}7.2 & \text { Trabalhos futuros } & 90\end{array}$

\begin{tabular}{ll}
\hline Referências Bibliográficas & 92
\end{tabular} 


\section{Lista de figuras}

1.1 Modelos Massivos. 13

2.1 Problema com o algoritmo do pintor. 18

\begin{tabular}{lll}
\hline 2.2 & Técnicas de culling. & 19
\end{tabular}

2.3 Pipeline do OpenGL. 22

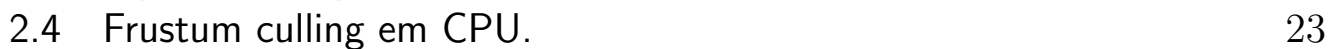

2.5 Frustum culling em GPU. 24

3.1 Tipos de Projeção. 26

3.2 Pseudo-código de descarte de pontos. 28

3.3 Volumes envolventes, imagem divulgada por Ericson et al. [23] 29

3.4 Teste de descarte de AABB. 30

3.5 Classificação de um ponto $\mathrm{P}$ contra o frustum. 31

3.6 Teste de pontos contra radar. 31

3.7 Teste de AABB contra radar. 32

3.8 Hierarquia de volumes envolventes. 33

3.9 Classificação dos vértices $\mathrm{n}$ e p. $\quad 35$

3.10 Volume envolvente do frustum. 36

3.11 Teste de descarte com apenas quatro planos. 37

3.12 Escape index. 38

3.13 SISD X SIMD. $\quad 40$

3.14 Dados utilizados no nó da hierarquia. 43

3.15 Aplicação desenvolvida para os testes. 44

3.16 Modelos com informações paramétricas. 45

3.17 Caminhos de câmera pelas plataformas. 46

3.18 Caminho de câmera pelo Boeing (73 segundos). $\quad 47$

4.1 Caminhos de câmera sem hierarquia nas plataformas. 50

4.2 Caminhos de câmera sem hierarquia no Boeing. 51

4.3 Caminhos de câmera com hierarquias onde média foi melhor. 53

4.4 Caminho de câmera com hierarquias onde mediana foi melhor. 54

4.5 Caminho de câmera sem pilha nas plataformas. 55

4.6 Caminho de câmera sem pilha no Boeing. 55

4.7 Otimizações sugeridas por Assarsoon nas plataformas. 57

4.8 Otimizações sugeridas por Assarsoon no Boeing. 57

4.9 Caminhos de câmera pelas plataformas com otimizações. 58

4.10 Caminhos de câmera pelo Boeing com otimizações. 59

4.11 Resultados obtidos por Assarsson em duas cenas. 61

4.12 Percurso com multithread. 61

4.13 Multiprocessamento sem troca de tarefas entre threads. 62

4.14 Multiprocessamento sem troca de tarefas entre threads no Boeing. 62

4.15 Multiprocessamento com troca de tarefas entre threads nas plataformas. 63

4.16 Multiprocessamento com troca de tarefas entre threads no Boeing. 64

4.17 Pipeline final em CPU. 64 
5.1 Evolução das placas gráficas até a quarta geração. 66

5.2 Evolução das placas gráficas a partir da quarta geração. 66

$\begin{array}{lll}5.3 & \text { Pipeline das placas gráficas modernas. } & 67\end{array}$

5.4 Variáveis do vertex shader das gpu primitives (extraída de [70]). $\quad 68$

5.5 Variáveis do pixel shader das gpu primitives (extraída de [70]). 68

5.6 Cilindro Gpu primitives. $\quad 69$

$\begin{array}{lll}5.7 & \text { Cone GPU primitives. } & 70\end{array}$

$\begin{array}{lll}5.8 & \text { Torus slice GPU primitives. } & 70\end{array}$

5.9 Frustum culling junto com GPU primitives.

5.10 Frustum culling em shader separado. $\quad 72$

5.11 Frustum culling em modelos genéricos. $\quad 74$

5.12 Frustum culling em modelos genéricos. $\quad 74$

5.13 Memória utilizada no percurso em GPU. 76

$\begin{array}{lll}5.14 & \text { Frustum culling nas GPU primitives. } & 77\end{array}$

5.15 Frustum culling em GPU para modelos genéricos. 77

6.1 Melhores caminhos de câmera em CPU e GPU nas plataformas. $\quad 80$

6.2 Melhores caminhos de câmera em CPU e GPU no Boeing. 81

\begin{tabular}{lll}
\hline 6.3 & Esquema do algoritmo de frustum culling híbrido. & 83
\end{tabular}

6.4 Possíveis estados do frustum culling híbrido. 84

6.5 Resultados do frustum culling híbrido nas plataformas. 86

6.6 $\quad$ Resultados do frustum culling híbrido no Boeing.

6.7 Resultados do frustum culling híbrido com render ligado. 88 


\section{Lista de tabelas}

2.1 Tabela comparativa de alguns algoritmos de visibilidade. 25

3.1 Modelos paramétricos. 45

3.2 Modelos com malhas triangulares. 46

4.1 Radar X Planos. 49

4.2 Radar sem interseção X Radar com interseção para o Boeing. 50

4.3 Hierarquias utilizando mediana. $\quad 52$

4.4 Hierarquias utilizando média. $\quad 52$

4.5 Detalhes dos caminhos com hierarquias de média e mediana. 54

4.6 Detalhes dos caminhos com hierarquias de média e mediana. 54

4.7 Resultados obtidos no trabalho de Assarsson. 56

4.8 Melhor combinação de otimizações utilizadas em cada modelo. 59

4.9 SIMD nos cálculos de descarte da P-43. 60

$5.1 \quad$ Memória necessária para os volumes envolventes.

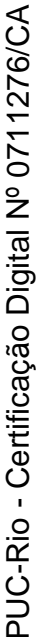

6.1 Comparação dos resultados entre melhor algoritmo em CPU e Híbrido. 87 
"A verdadeira felicidade está na própria casa, entre as alegrias da família."

Leon Tolstoi, Leon Tolstoi. 